\title{
On Quality of Usage Provisioning for Electricity Scheduling in Microgrids
}

\author{
Yingsong Huang, Student Member, IEEE, and Shiwen Mao, Senior Member, IEEE
}

\begin{abstract}
Microgrid is a promising component for future smart grid deployment. The balance of supply and demand of electricity is one of the most important requirements of microgrid management. In this paper, we investigate the grid stability problem from an admission control perspective, while guaranteeing the quality of usage (QoU) of local residents in a microgrid under both random electricity supply and demand. In particular, a QoU request is generated when the electricity demand exceeds the microgrid supply. The microgrid control center aims to maintain the QoU blocking probability around a target value by serving (i.e., switching to the macrogrid for extra electricity supply) or blocking QoU requests. The problem is formulated as a queue stability problem by introducing the concept of a QoU blocking virtual queue. Lyapunov optimization is then applied to derive an adaptive QoU scheduling algorithm with low complexity $\mathcal{O}(1)$. Furthermore, it is an online algorithm since it does not require any future knowledge of the electricity supply and demand processes. The stability of the algorithm is proven, and its performance is evaluated with trace-driven simulations under random or nonstationary QoU requests. The simulation results demonstrate the efficacy and robustness of the proposed QoU scheduling algorithm.
\end{abstract}

Index Terms-Distributed renewable energy resource (DRER), Lyapunov optimization, macrogrid, microgrid, quality of usage (QoU), smart grid, stability.

\section{NOMENCLATURE}

$\begin{array}{ll}\text { Symbol } & \text { Definition } \\ D(t) & \text { Total electricity demand at time slot } t . \\ S(t) & \text { Total electricity supply a time slot } t . \\ \alpha(t) & \text { QoU request at time slot } t \text {. } \\ E_{\max } & \text { Maximum allowable QoU requests. } \\ \delta & \text { Target QoU blocking probability. } \\ p & \text { Target average QoU blocking rate. } \\ \lambda & \text { Average QoU request arrival rate. } \\ J(m) & \text { The MGCC decision for QoU unit } m . \\ I(t) & \text { QoU scheduling decision at time slot } t . \\ I_{a}(t) & \text { Automatic blocking decision for } D(t)-S(t) \geq \\ Z(t) & E_{\text {max. }} \\ L(\cdot) & \text { Backlog of the QoU blocking virtual queue. } \\ \Delta(Z(t)) & \text { Lyapunov function. }\end{array}$

Manuscript received April 15, 2012; revised September 29, 2012; accepted January 23, 2013. Date of publication June 26, 2013; date of current version May 22, 2014. This work was supported in part by the U.S. National Science Foundation (NSF) under Grant CNS-0953513 and in part by the NSF Broadband Wireless Access and Applications Center at Auburn University.

The authors are with the Department of Electrical and Computer Engineering, Auburn University, Auburn, AL 36839-5201 USA (e-mail: yzh0002@ tigermail.auburn.edu; smao@ieee.org).

Color versions of one or more of the figures in this paper are available online at http://ieeexplore.ieee.org.

Digital Object Identifier 10.1109/JSYST.2013.2260941
$B \quad$ A constant that is $\frac{1}{2}\left(1+\delta^{2}\right)$.

$\epsilon \quad$ A positive constant.

\section{INTRODUCTION}

N MART GRID is an electricity network, a 21st century evolution of the utility electricity delivery systems. Smart grid enhances the traditional power grid through computing, communications, and control technologies throughout the processes of electricity generation, transmission, distribution, and consumption. The two-way flow of electricity and real-time information is a characteristic feature of smart grid, which offers many technical benefits and flexibilities to both utility providers and consumers. It balances the supply and demand in a timely fashion and improves the energy efficiency and electricity grid stability. According to the U.S. 2009 Recovery Act [1], a smart grid will replace the traditional system and is expected to save consumer's cost and reduce America's dependence on foreign oil, which is achieved by improving efficiency and spurring the use of renewable energy sources.

Microgrid is a promising component for future smart grid deployment. Due to the increasing deployment of distributed renewable energy resources (DRERs), microgrid provides a localized cluster of renewable energy generation, storage, distribution, local demand, and consumption, to achieve reliable and effective energy supply with simplified implementation of smart grid functionalities [2], [3]. A typical structure of microgrid is illustrated in Fig. 1, which includes one or more renewable energy sources (such as wind turbines and solar panels), wireless networks for information delivery, a microgrid central controller (MGCC), local residents, and commercial entities. Some microgrids also have storage capability for storing excess electricity for future use. The microgrid is centrally controlled and managed by the MGCC [3], which exchanges information with both energy sources and local residents via a two-way information network, such as a wireless network [4] or a power line communication system [5]. There is a single common coupling point between the microgrid and the macrogrid, which can be switched on or off. When switched off, the microgrid is disconnected from the macrogrid and works in the islanded mode, in which the DRERs continuously provide electricity to the local residents. When switched on, the microgrid is connected to the macrogrid and may request extra electricity from the macrogrid or sell the excess energy back to the market [6].

The balance of supply and demand of electricity energy is one of the most important requirements for microgrid management [2]. In this paper, we investigate the problem of 


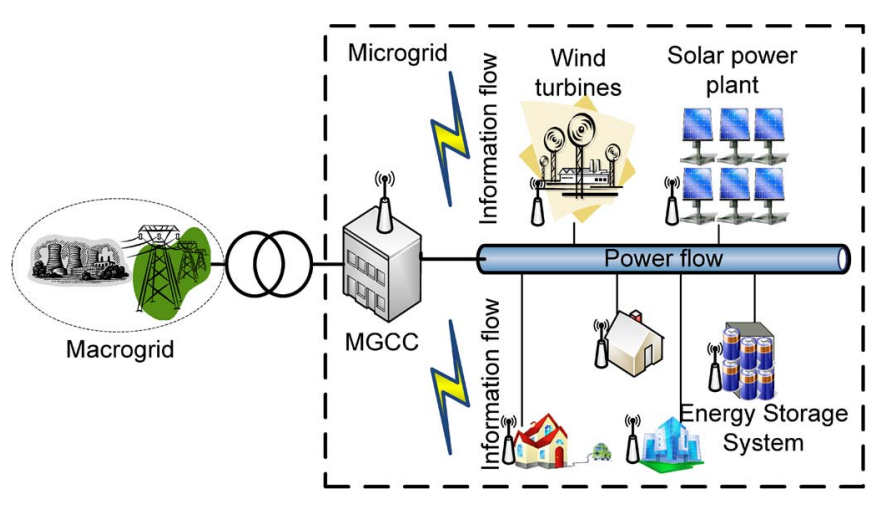

Fig. 1. Illustration of the microgrid architecture considered in this paper.

guaranteeing the residents' quality of usage (QoU) of electricity in a microgrid. In practice, the electricity usage of a user can be classified into two categories: priority load that must be satisfied by the basic capacity design of the microgrid and excessive load that can be deferred or declined. Users may set the load amount for each type according to their preferences [7]. The QoU of residents can be defined as the satisfaction level of the excessive electricity demand for more comfortable life, such as excessive use of air conditioners and entertainment devices [8].

We design an adaptive MGCC control policy to guarantee the residents' satisfactory ratio and maintain the stability of the grid. The electricity generated from renewable sources is generally random, due to complex weather conditions, while the electricity demand is also random due to random consumer behavior. It may be challenging to identify the exact distributions of supply and demand for microgrid management, since the weather process is usually found to be self-similar [9] and the consumer demand may be nonstationary. It may also be very costly to have precise real-time monitoring of the supply and demand processes. Therefore, a simple low-cost scheme that does not rely on any statistical information of the supply and demand processes would be highly desirable.

In this paper, we tackle the problem of QoU provisioning with a Lyapunov optimization approach, which is a useful technique to solve stochastic optimization and stability problems [10], [11]. We first introduce the concept of a QoU blocking virtual queue to transform the QoU scheduling problem to a queue stability problem. Second, we design an adaptive $\mathrm{QoU}$ control algorithm based on the Lyapunov optimization method and prove that the algorithm stabilizes the blocking virtual queue, which is equivalent to ensuring both the QoU satisfaction ratio of residents and grid stability. The proposed algorithm has low computation complexity, i.e., $\mathcal{O}(1)$. Furthermore, it can be implemented online because it only relies on the current system status; it does not require any further knowledge of the electricity demand and supply processes. The proposed algorithm also converges exponentially due to the nice property of Lyapunov stability design [12]. The algorithm is evaluated with trace-driven simulations and is shown to achieve QoU stability with fast convergence and robustness.

The remainder of this paper is organized as follows. We present the microgrid QoU stability system model and transform the problem to a queue stability problem in Section II. An adaptive microgrid QoU scheduling algorithm is developed, and its stability is proven in Section III. Simulation results are presented and discussed in Section IV. We discuss related work in Section V. Section VI concludes this paper.

\section{System Model}

We consider the residential electricity consumption in a microgrid. Specifically, we assume that there is one or more DRERs in the microgrid, which may consist of wind turbines and solar panels. We further assume that the microgrid is designed such that a portion of the electricity demand related to the basic living usage (e.g., lighting and refrigerator usage) can be guaranteed by the basic capacity of the DRERs. There is randomness in both electricity supply (e.g., due to weather or wind speed changes) and demand (e.g., more entertainmentrelated usage in weekends). To cope with the randomness, the microgrid works in the grid-connected mode. The residents send their demands, and the DRERs send their supplies to the MGCC via the information network (such as a power line communication network or a wireless network). The MGCC then accumulates the total demand and supply based on the received information.

The MGCC identifies a $Q o U$ request when the total demand exceeds the supply, which consists of the difference between the total requested energy and the energy supplied by the DRERs. Greedily satisfying all the excessive QoU requests, i.e., by purchasing electricity from the macrogrid, may cause large load fluctuations to the macrogrid and be harmful to grid stability. Such a strategy is also not economically efficient for the operation of the microgrid, due to utility market price fluctuations. Therefore, we consider an MGCC operation mechanism that determines the amount of QoU requests that need to be satisfied by purchasing extra electricity from the macrogrid, while declining the rest of the requests.

After MGCC aggregates the demand profiles through the information network and applies the proposed admission control, the control commands are then transmitted to the users via the communication network and then reach the smart loads and smart appliances via home area networks (HANs). The smart meters provide the interface between the communication network and HAN and serve as the gateway for security authentication and command interpretation to ensure the command issued from the trusted MGCC. Then, the smart meters plan the service level of each smart load/facility and send out the control commands with the service level of individual loads through the HAN. After the smart load obtains the command, it then adjusts its level to conform to the new allocation [13].

We assume that the excess electricity usage can tolerate a prescribed QoU blocking probability. For example, some excess air conditioning cooling- or heating-related $\mathrm{QoU}$ requests could be declined, leading to electricity savings. The MGCC adaptively serves or blocks QoU requests to maintain a prescribed QoU blocking probability as well as stability of the grid, as shown in Fig. 2. Specifically, when a QoU request is triggered, the MGCC can choose to satisfy this demand with a predetermined probability $1-\delta$ or block it with average blocking probability $\delta$. The practical outage risk of the microgrid under the admission control mechanism can be designed through insurance 


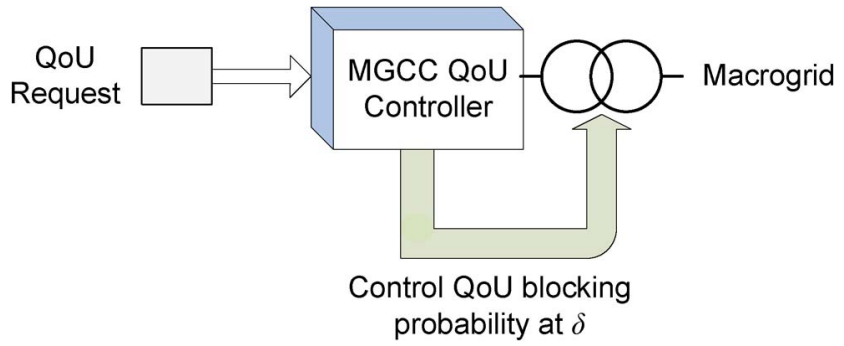

Fig. 2. System model considered in this paper.
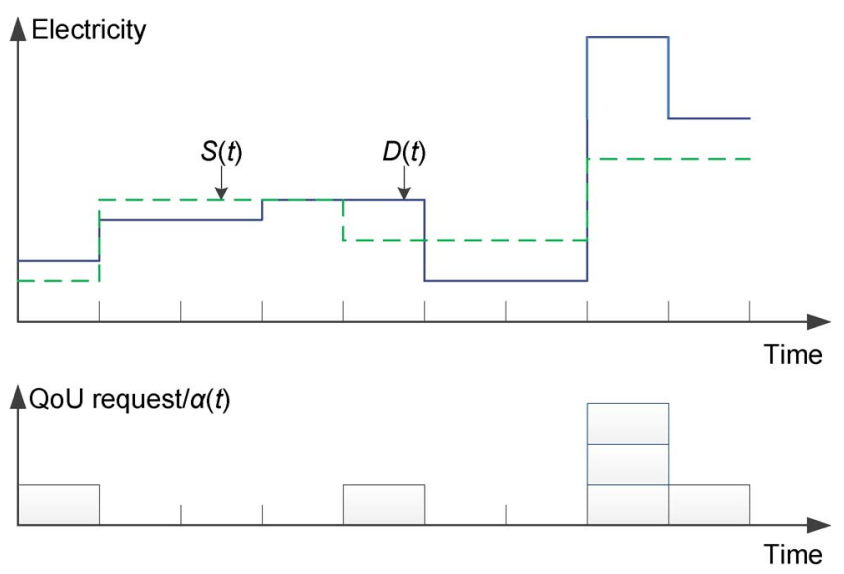

Fig. 3. Example of supply $S(t)$, demand $D(t)$, and QoU requests $\alpha(t)$.

premium [14]. Assuming that the average QoU request arrival rate is $\lambda$, the average blocking rate for the QoU requests can be expressed as $p=\delta \cdot \lambda$. If the MGCC serves the request, it will switch on the coupling point to connect to the macrogrid and request extra electricity from the nonrenewable energy sources in the macrogrid. Otherwise, the QoU request will be blocked, and the consumer will not be able to get the electricity for the desired task.

We consider the electricity demand and supply on a daily basis, which consists of time slots [15]. Without loss of generality, we assume that the one-day period is slotted into $T$ time slots, each of which has a duration that is determined by the timescale of the demand and supply, as well as how frequent the microgrid can switch on and off to the macrogrid. Let the total residential demand at time slot $t$ be $D(t)$ and the total supply from the DRERs be $S(t)$, each being a random process varying from time slot to time slot. As shown in Fig. 3, when $D(t)$ is smaller than $S(t)$, the demand will all be satisfied with the local supply. Without loss of generality, we assume that the excess supply in a time slot $t$ can be stored [i.e., accumulated into $D(t+1)$ ] and used to accommodate future QoU requests. When $D(t)$ exceeds $S(t)$ in a time slot $t$, the local storage and supply are insufficient to serve the demand, and a QoU request will be generated.

Let $\alpha(t)$ be the function representing the arrival process of QoU requests, as

$$
\alpha(t)=\min \left\{[D(t)-S(t)]^{+}, E_{\max }\right\} .
$$

At each time slot $t, \alpha(t)$ takes values in a finite set $\left\{0,1, \ldots, E_{\max }\right\}$, in certain basic unit of electricity usage. In (1), $E_{\max }$ is the maximum amount of electricity that can be requested from the macrogrid in one time slot.

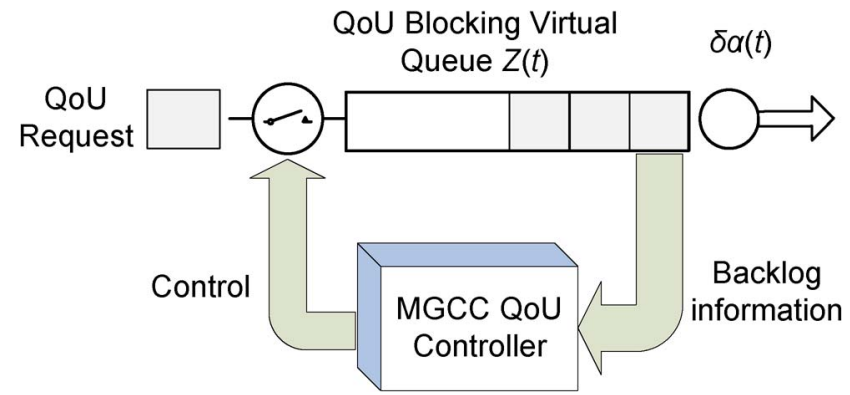

Fig. 4. QoU blocking virtual queue model.

Then, the average QoU request arrival rate is

$$
\lambda=\lim _{t \rightarrow \infty} \frac{1}{t} \sum_{\tau=0}^{t-1} \alpha(\tau) .
$$

In (1), the upper limit $E_{\max }$ is due to the practical constraints such as power line transmission, substation capacity, and power procurement cost on the energy market. The microgrid may not support an excessive energy use if the QoU request exceeds a prescribed threshold, even when it is switched on to the macrogrid. An automatic blocking operation will be applied to decline the excess parts that are above $E_{\max }$. This automatic blocking operation serves as a disturbance to the microgrid system, which should be addressed in the system stability design. The scheduling policy at the MGCC is then designed to stabilize the system under the presence of such disturbances.

At every time slot $t$, the MGCC scheduler takes an action in the set $\{0$ : serve, 1 : block $\}$ for each incoming QoU request unit. Supposing that $\alpha(t)=M$, the MGCC operates on each of the $M$ QoU units as

$J(m)= \begin{cases}1, & \text { QoU request unit } m \text { is blocked } \\ 0, & \text { otherwise }\end{cases}$

$$
m=1,2, \ldots, M \text {. }
$$

Then, we have $I(t)=\sum_{m=1}^{M} J(m)$ for time slot $t$. The average blocking rate can be evaluated as

$$
p=\lim _{t \rightarrow \infty} \frac{1}{t} \sum_{\tau=0}^{t-1} I(\tau) .
$$

The microgrid adopts the scheduling policy $I(t)$ to maintain the stability of the system and keeps the average QoU blocking rate at a prescribed level $p=\delta \cdot \lambda$.

We next introduce a conceptual $Q o U$ blocking virtual queиe $Z(t)$, as shown in Fig. 4. The queue is "virtual" because it is maintained by the MGCC control algorithm, without network traffic arrivals and departures. It is used to track the number of the blocking events resulted from the MGCC control actions [16]. When a QoU request unit is granted, the queue length will be increased by one; the queue backlogs will be depleted at a rate of $\delta \cdot \alpha(t)$ at time slot $t$. The MGCC scheduler enqueues or drops incoming QoU requests according to policy $I(t)$ at each time slot $t$. It is worth noting that, unlike an actual queue, the virtual queue backlog $Z(t)$ may take negative values. 
The system equation that governs the dynamics of the QoU blocking virtual queue is

$$
Z(t+1)=Z(t)-\delta \cdot \alpha(t)+I(t) .
$$

We then have the following proposition that establishes the equivalence of maintaining the stability of the QoU blocking virtual queue and QoU assurance.

Proposition 1: If an MGCC control policy $I(t)$ stabilizes the QoU blocking virtual queue $Z(t)$, the microgrid will be stabilized at the average QoU blocking rate $p=\delta \cdot \lambda$.

Proof: According to the dynamics of the QoU blocking virtual queue (5), we have

$$
\begin{aligned}
Z(1)= & Z(0)-\delta \cdot \alpha(0)+I(0) \\
Z(2)= & Z(1)-\delta \cdot \alpha(1)+I(1) \\
& \cdots \\
Z(t)= & Z(t-1)-\delta \cdot \alpha(t-1)+I(t-1) .
\end{aligned}
$$

Summing up both sides of (6), we obtain

$$
Z(t)=Z(0)-\delta \cdot \sum_{\tau=0}^{t-1} \alpha(\tau)+\sum_{\tau=0}^{t-1} I(\tau) .
$$

Dividing (7) by $t$ and then taking $\operatorname{limit}_{\lim } \lim _{t \rightarrow \infty}$ on both sides, we have

$$
\lim _{t \rightarrow \infty} \frac{Z(t)-Z(0)}{t}=\lim _{t \rightarrow \infty} \frac{1}{t}\left[-\delta \cdot \sum_{\tau=0}^{t-1} \alpha(\tau)+\sum_{\tau=0}^{t-1} I(\tau)\right] .
$$

If the virtual queue is rate stable by a control policy $I(t)$, the backlog $Z(t)$ should be finite. We have $\lim _{t \rightarrow \infty}(1 / t)[Z(t)-$ $Z(0)]=0$, which yields

$$
\lim _{t \rightarrow \infty} \frac{1}{t} \sum_{\tau=0}^{t-1} I(\tau)=\delta \cdot \lim _{t \rightarrow \infty} \frac{1}{t} \sum_{\tau=0}^{t-1} \alpha(\tau) \Rightarrow p=\delta \cdot \lambda
$$

according to definitions (2) and (4).

Proposition 1 transforms the QoU control problem into a queue stability problem, which can be solved with a system stability design from the control theory point of view. Accordingly, we next derive the control policy by applying the Lyapunov optimization method to stabilize the virtual queue $Z(t)$, thus solving the microgrid QoU scheduling problem.

\section{Adaptive Microgrid QoU CONTROL Policy}

With Proposition 1, we show that the QoU control problem is equivalent to the virtual queue stability problem. We then use the Lyapunov optimization technique to develop an adaptive QoU control algorithm. This algorithm is simple to implement and does not require a priori statistical knowledge of the electricity supply or demand processes and is also robust to non independent and identically distributed (i.i.d.) and nonergodic behaviors [11].

\section{A. Lyapunov Optimization}

In Lyapunov stability analysis, a Lyapunov function

$$
L(\cdot): \mathbb{R}^{n} \rightarrow \mathbb{R}
$$

is an energylike positive definite function [17]. Roughly speaking, for a network system, if we can find a suitable Lyapunov function and let the control decision at every time slot $t$ greedily minimize the "drift" (will be defined hereinafter shortly), then the network state will be consistently pushed toward lowbacklog states, and the network stability will be thus maintained [10], [11].

We define the following Lyapunov function:

$$
L(Z(t))=\frac{1}{2}[Z(t)]^{2}
$$

which is positive definite when $Z(t) \neq 0$, and $L(Z(t))=0$ if and only if $Z(t)=0$. Intuitively, the Lyapunov function represents the energy of the dynamic system governed by (5). Without loss of generality, we first assume that $E_{\max }=1$ for the following analysis to derive the QoU scheduling policy. For the general case of $E_{\max }>1$, we can apply the scheduling policy for each of the QoU units.

We define the conditional one-slot Lyapunov drift as

$$
\Delta(Z(t))=\mathbb{E}\{L(Z(t+1))-L(Z(t)) \mid Z(t)\} .
$$

With some algebraic manipulation, we have from (10)

$$
\begin{aligned}
\Delta(Z(t))= & \frac{1}{2} \mathbb{E}\left\{\left[I(t)^{2}+(\delta \cdot \alpha(t))^{2}-2 \delta \cdot \alpha(t) \cdot I(t)\right]\right. \\
& +2 Z(t)(I(t)-\delta \cdot \alpha(t)) \mid Z(t)\} \\
\leq & \frac{1}{2} \mathbb{E}\left\{\left(I(t)^{2}+(\delta \cdot \alpha(t))^{2}\right) \mid Z(t)\right\} \\
& +\mathbb{E}\{Z(t)(I(t)-\delta \cdot \alpha(t)) \mid Z(t)\} \\
\leq & \frac{1}{2}\left(1+\delta^{2}\right)-Z(t) \mathbb{E}\{\delta \cdot \alpha(t)\} \\
& +\mathbb{E}\{Z(t) I(t) \mid Z(t)\} \\
= & B-Z(t) \cdot \delta \cdot \lambda+\mathbb{E}\{Z(t) I(t) \mid Z(t)\}
\end{aligned}
$$

where $B=(1 / 2)\left(1+\delta^{2}\right)$ is a constant. The first inequality is because $\delta, \alpha(t)$, and $I(t)$ are all nonnegative. The second inequality is due to the fact that $I(t) \leq 1$.

Note that, in the drift (11), the QoU control decision $I(t)$ only affects the last term on the right-hand side (RHS). We can design an algorithm to minimize the upper bound of the drift by observing the current virtual queue state $Z(t)$, i.e., minimizing the RHS of (11) at every time slot $t$. Such an approach leads to the following optimization problem:

$$
\min _{I(t)}\{Z(t) I(t)\}, \quad \text { for } t=1,2, \ldots
$$

Solving the aforementioned minimization problem at each time slot $t$, we obtain the QoU scheduling policy $I(t)$. It can be seen from (11) that the drift will be negative when $Z(t)$ is sufficiently 
large. Thus, the stability of the virtual queue is ensured. We find that this problem has a straightforward threshold-based solution, as given in Section III-B.

\section{B. Adaptive Microgrid QoU Control Policy}

We can solve problem (12) by observing the current virtual queue backlog $Z(t)$. If $E_{\max }=1$, this is the situation in which, at most, one unit QoU request arrives in a time slot. The following adaptive QoU control policy can be obtained:

$$
I(t)=\left\{\begin{array}{ll}
0, & Z(t) \geq 0 \\
1, & Z(t)<0
\end{array} \quad \text { for } t=1,2, \ldots\right.
$$

which is a solution to problem (12). Specifically, if the current virtual queue backlog $Z(t) \geq 0$, the MGCC will choose $I(t)=$ 0 , i.e., to serve the $\mathrm{QoU}$ request. Otherwise, if $Z(t)<0$, the MGCC will choose $I(t)=1$, i.e., to reject the QoU request. After the decision is made, the QoU blocking virtual queue $Z(t)$ will be updated as given in the system (5).

Recall that the parts exceeding $E_{\max }$ in a QoU request will be automatically blocked, even if the scheduling policy $I(t)=0$. Such an automatic blocking decision is denoted as $I_{a}(t)=1$. In this case, the virtual queue will be increased by $\max \left\{I(t), I_{a}(t)\right\}$. This can be interpreted as a disturbance at time $t$ in the control system. However, with policy (13), the Lyapunov drift will still be pushed toward the minimum value and keeps the system stable.

It can be noted that this algorithm actually solves a minimum weight matching problem, where weight is defined to be the virtual queue backlog $Z(t)$ [18]. Furthermore, the algorithm only requires the current virtual queue backlog information: It neither requires any knowledge on future DRER supply and demand nor requires any knowledge on the statistics of the supply and demand processes. Therefore, this is an online algorithm that can be easily implemented, with computational complexity $\mathcal{O}(1)$. The detailed adaptive QoU scheduling algorithm is presented in Algorithm 1.

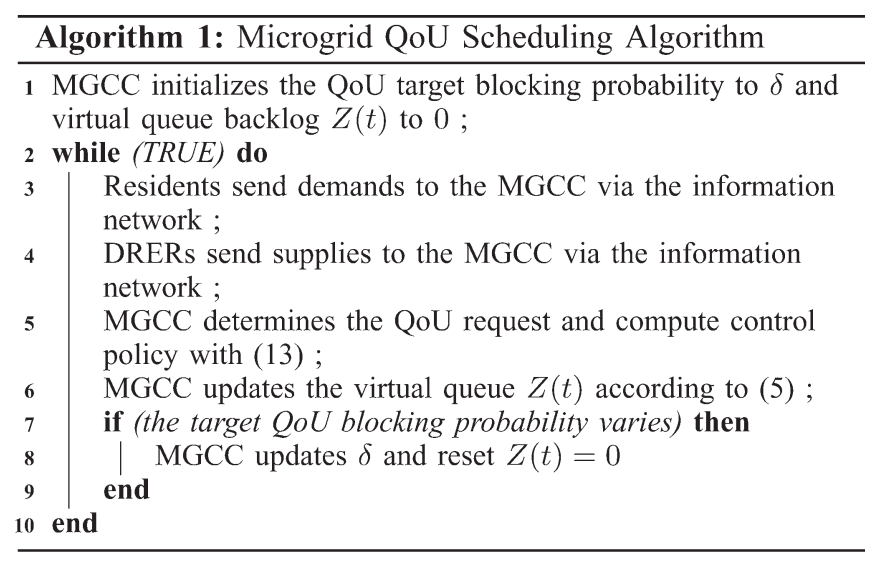

For the general case of $E_{\max }>1$, the QoU scheduling algorithm operates on each unit of the total QoU request in the time slot. For example, if $\alpha(t)=l$, then the algorithm divides the time slot $t$ into $l$ stages, applies (13) to find the scheduling policy for each QoU request unit, and updates the QoU blocking virtual queue in each stage.
We have the following proposition on the performance of the microgrid QoU scheduling algorithm given in Algorithm 1.

Proposition 2: The adaptive microgrid QoU scheduling policy given in Algorithm 1 stabilizes the microgrid system. Furthermore, the average backlog of the QoU blocking virtual queue is upper bounded by $B / \epsilon$, where $B=(1 / 2)\left(1+\delta^{2}\right)$ and $\epsilon>0$ are constant.

Proof: Let $\hat{I}(t)$ be a policy other than the proposed control policy $I(t)$ given in (13). With the drift bound (11) and the fact that $I(t)$ minimizes the drift bound (11), we have

$$
\begin{aligned}
\Delta(Z(t)) & \leq B-Z(t)(\delta \cdot \lambda-\mathbb{E}\{I(t) \mid Z(t)\}) \\
& \leq B-Z(t)(\delta \cdot \lambda-\mathbb{E}\{\hat{I}(t) \mid Z(t)\}) .
\end{aligned}
$$

We can choose $\hat{I}(t)$ to satisfy the following two conditions:

$$
\left\{\begin{array}{ll}
\delta \cdot \lambda-\mathbb{E}\{\hat{I}(t) \mid Z(t)\}=\epsilon, & \text { if } Z(t) \geq 0 \\
\delta \cdot \lambda-\mathbb{E}\{\hat{I}(t) \mid Z(t)\}=-\epsilon, & \text { if } Z(t)<0
\end{array} \text { for } t=1,2, \ldots\right.
$$

where $\epsilon>0$ is a constant.

It follows (14) that the drift achieved by policy $I(t)$ given in (13) satisfies

$$
\Delta(Z(t)) \leq B-\epsilon \cdot|Z(t)| .
$$

Taking expectation on both sides of (15), we have

$$
\mathbb{E}\{\Delta(Z(t))\} \leq B-\epsilon \cdot \mathbb{E}\{|Z(t)|\} .
$$

According to the drift definition, we have

$\mathbb{E}\{L(Z(t+1)) \mid Z(t)\}-\mathbb{E}\{L(Z(t)) \mid Z(t)\} \leq B-\epsilon \cdot \mathbb{E}\{|Z(t)|\}$.

Summing up both sides of (16) over $K$ time slots $t \in[0, \ldots$, $K-1$ ], we have

$$
\begin{aligned}
\mathbb{E}\{L(Z(K)) \mid Z(t)\}-\mathbb{E} & \{L(Z(0)) \mid Z(t)\} \\
& \leq B \cdot K-\epsilon \cdot \sum_{t=0}^{K-1} \mathbb{E}\{|Z(t)|\} .
\end{aligned}
$$

Since the Lyapunov function is nonnegative, we have $\mathbb{E}\{L(Z(K)) \mid Z(t)\} \geq 0$. Removing this nonnegative term on the left-hand side (LHS) of (17), the inequality still holds true. We then divide both sides of (17) by $(\epsilon \cdot K)$ and take $\operatorname{limit} \lim _{K \rightarrow \infty}$. The LHS becomes zero since the initial state $\mathbb{E}\{L(Z(0)) \mid Z(t)\} \leq \infty$ is finite. We then obtain the following bound on the average virtual queue length:

$$
\lim _{K \rightarrow \infty} \frac{1}{K} \sum_{t=0}^{K-1} \mathbb{E}\{|Z(t)|\} \leq B / \epsilon .
$$

Thus, the system is mean rate stable, and the average backlogs of the queue are upper bounded by $B / \epsilon$.

\section{Simulation Study}

We demonstrate the performance of the proposed adaptive QoU control policy through extensive simulations. We simulate a microgrid with 25 residents, in which the residents' demands 


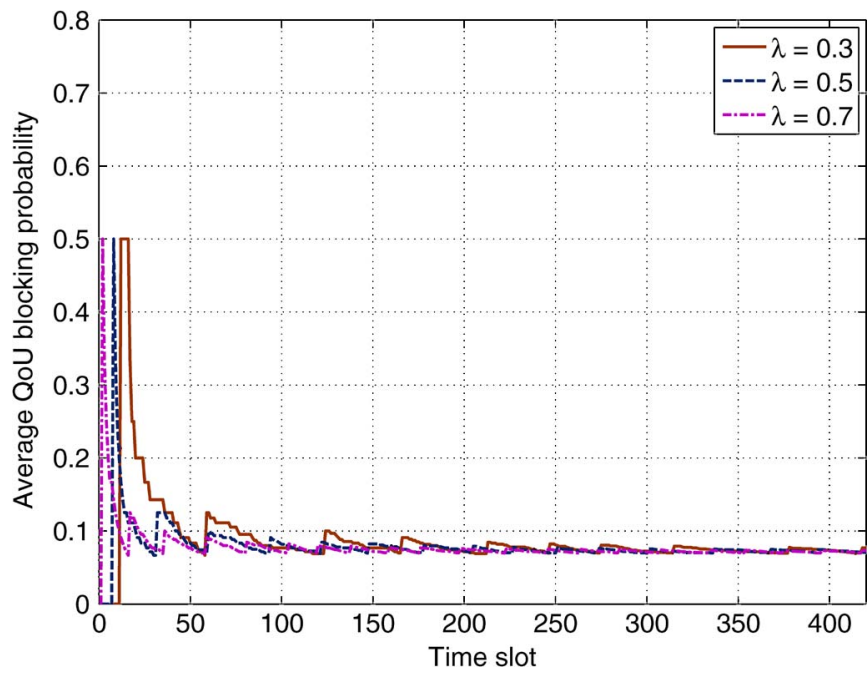

Fig. 5. Average QoU blocking probability with different QoU request rates.

are supplied by a wind turbine plant. We use the renewable energy supply data from the Western Wind Resources Dataset published by the National Renewable Energy Laboratory [19]. When a QoU request arrives, the MGCC either satisfies the QoU request by requesting electricity from the macrogrid or blocks this request according to the adaptive QoU control policy. We assume that the time slot duration is $10 \mathrm{~min}$ and the simulation duration is one week. The QoU blocking probability is set to $\delta=0.07$, and the maximum QoU is set to $E_{\max }=1$, unless otherwise specified.

We first investigate the average QoU blocking probability with the default parameter settings. We model QoU requests as i.i.d. process over time slots. The average QoU arrival rates are $\lambda=0.3,0.5$, and 0.7 for three simulations, respectively. We show the first 420 time slots in Fig. 5, corresponding to $70 \mathrm{~h}$. It can be seen that all the three average QoU blocking probabilities converge to the target probabilities after about 150, 90 , and 50 time slots, respectively. The higher the QoU request rate, the faster the convergence, since the algorithm is triggered by QoU arrivals and is executed more often for higher $\mathrm{QoU}$ request rate. The converged blocking probabilities are 0.0726 , 0.0711 , and 0.0704 , respectively, which are very close to the target blocking probability of 0.07 . It should be noted that the algorithm only relies on the current backlog of the virtual queue; it does not require any further statistical information of the demand and supply processes. Thus, the proposed QoU algorithm is effective and easy to implement.

We next examine the virtual queue backlog for the case of $\lambda=0.3$. In Fig. 6, we find that the algorithm attempts to push the instant backlog toward zero. The average queue length is stabilized around 0.4. The average queue length is bounded during the entire period with $\epsilon=0.5$, which ensures that the average blocking probability is stabilized around the target rate. Recall that the instant virtual backlog can take negative values, as discussed in Section II. For the cases of $\lambda=0.5$ and 0.7, similar results are observed but omitted for brevity.

We also explore the performance of the proposed algorithm under time-varying target QoU blocking rates, due to the different microgrid operation strategies at different time periods. We

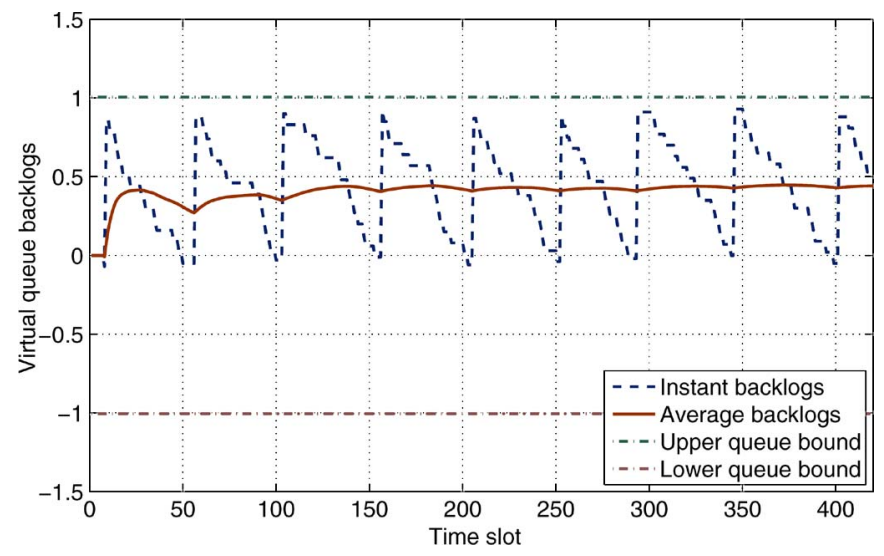

Fig. 6. Instant and average virtual queue backlogs when $\lambda=0.3$.

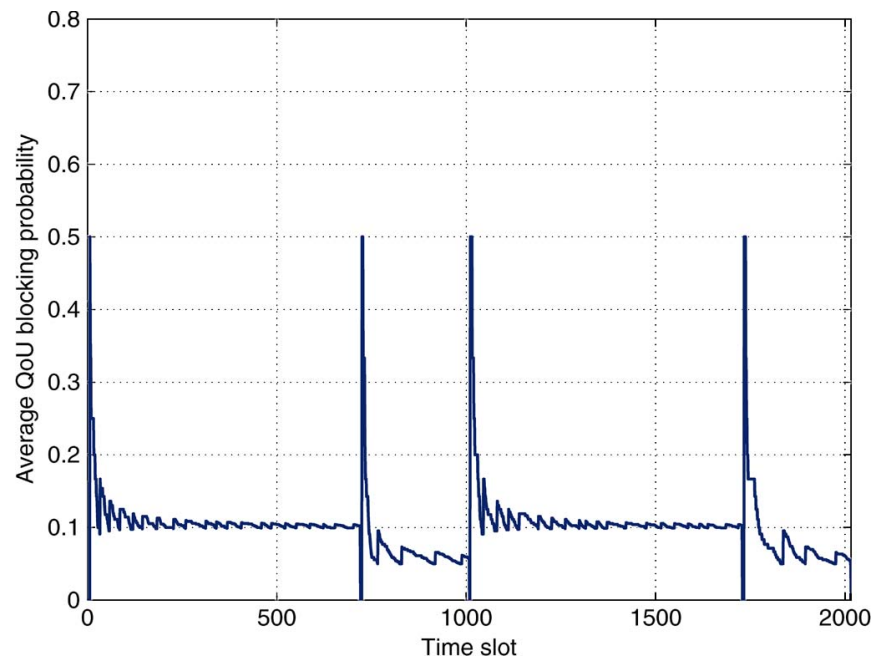

Fig. 7. Average QoU blocking probability with varying target QoU blocking rates.

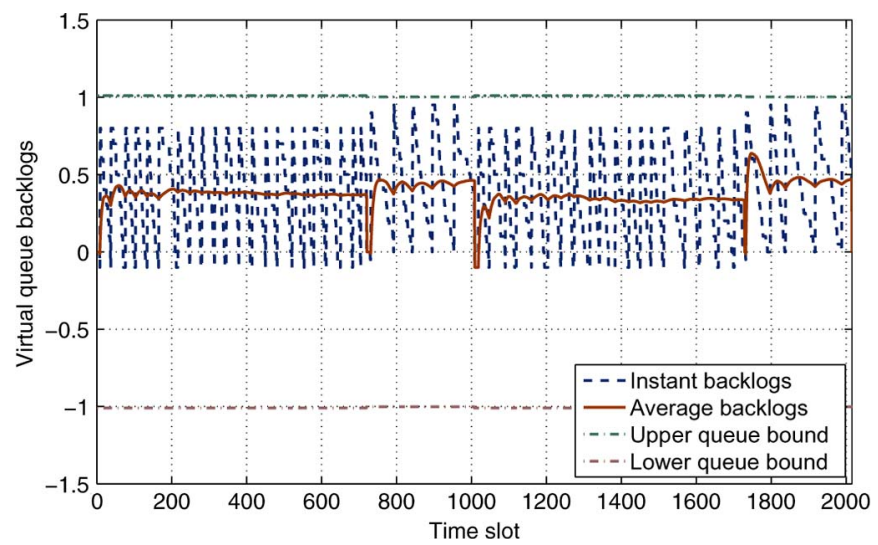

Fig. 8. Instant virtual queue backlogs with varying target QoU blocking rates.

assume that, from Monday to Friday, the blocking probability is set to 0.1 and, in the weekend, the blocking probability is changed to 0.05 to accommodate more entertainment-related electricity consumption. We simulate the QoU control policy for a two-week period. The average QoU blocking probability is plotted in Fig. 7, and the instant virtual queue backlog is plotted in Fig. 8. From the results, we observe that the algorithm keeps on tracking the target QoU blocking rate and converges 


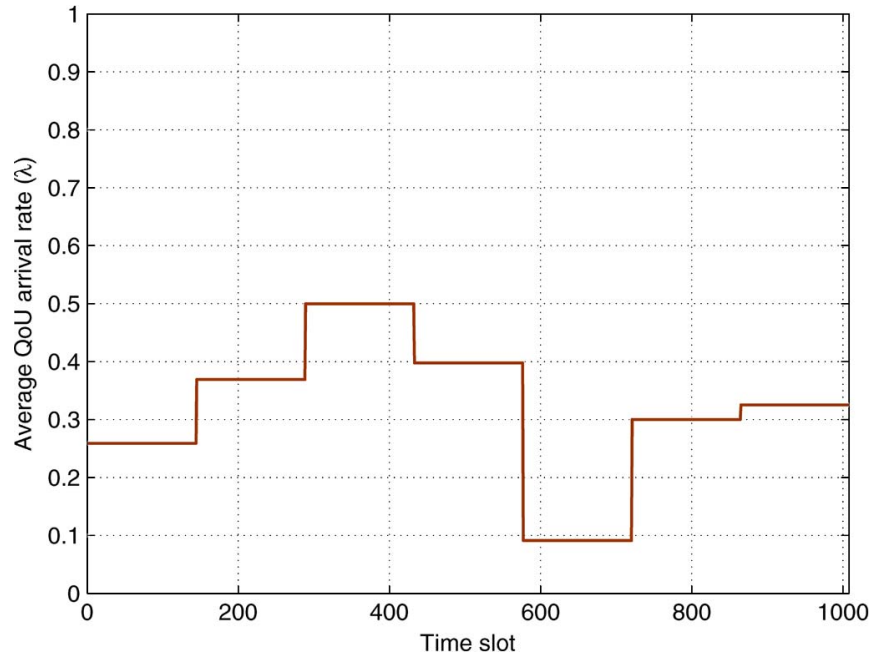

Fig. 9. Average QoU arrival rate with a normal distribution.

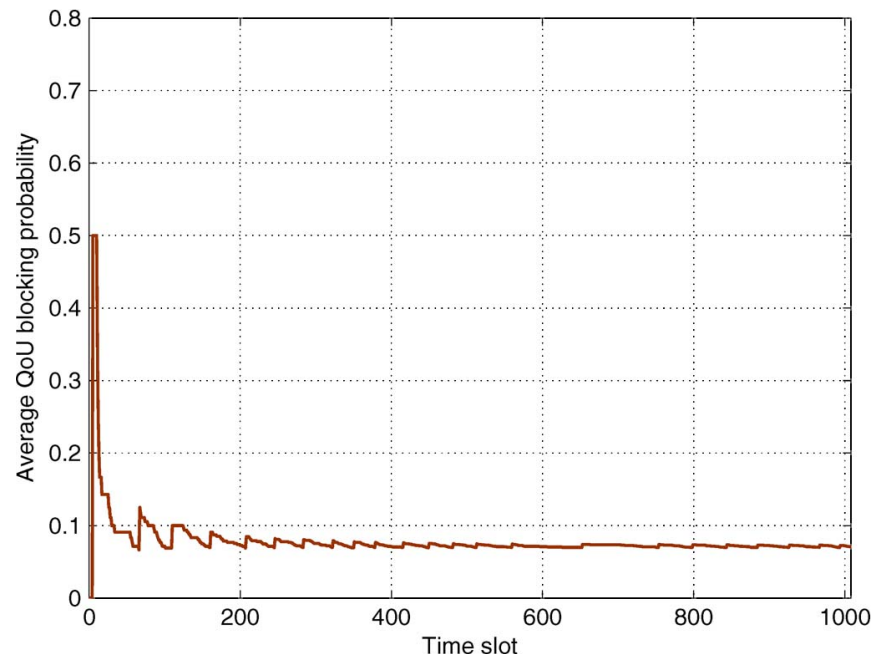

Fig. 10. Average QoU blocking probability with time-varying QoU arrival rates-normal distribution.

to around $0.1024,0.0575,0.1029$, and 0.0548 , respectively, and the average virtual queue is bounded.

Therefore, the proposed QoU policy is dynamically adaptive to different microgrid operation strategies. At the strategy switching point, there is very short period of deviation of the QoU blocking probability, due to the virtual queue reset for the new grid operation strategy. However, as time elapses, the average QoU blocking probability converges to the target rate very quickly. It is also interesting to see that, during the period of $\delta=0.05$, the backlogs evolve more slowly than that of $\delta=0.1$, because of the smaller service rate $\delta$ as given in the virtual queue dynamics (5).

We further evaluate the algorithm under time-varying $\mathrm{QoU}$ request rates. In these simulations, we use the default setting but let the mean QoU arrival rate be a random number following normal distribution with a mean rate of 0.3 and a standard deviation of 0.1 . The QoU arrival rate, blocking probability, and virtual queue backlog are plotted in Figs. 9, 10, and 11, respectively. We observe that the proposed algorithm stabilizes the system by keeping the QoU blocking rate around 0.07 . The virtual queue backlogs are mean rate stable as well.

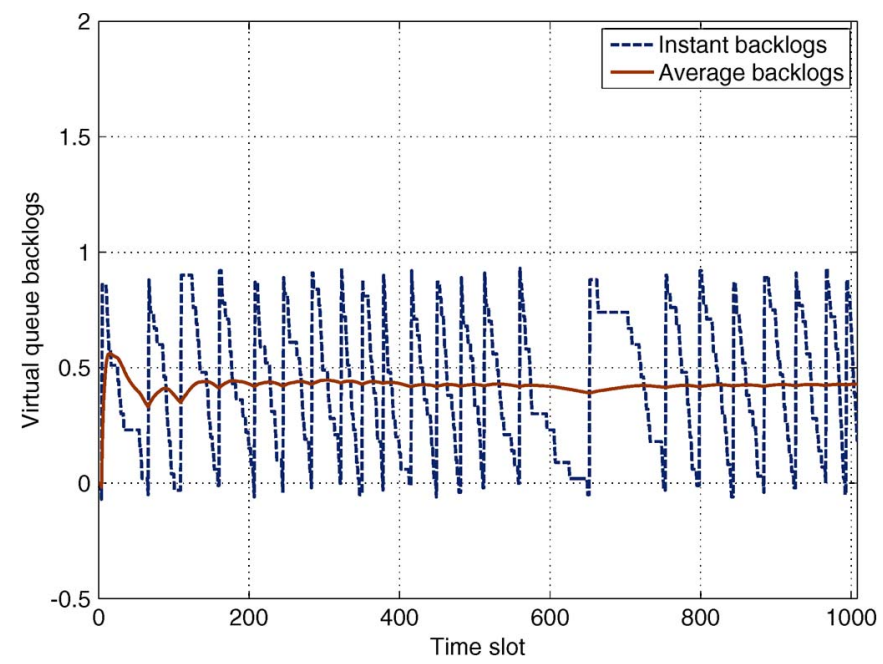

Fig. 11. Instant and average virtual queue backlogs with time-varying QoU arrival rates-normal distribution.

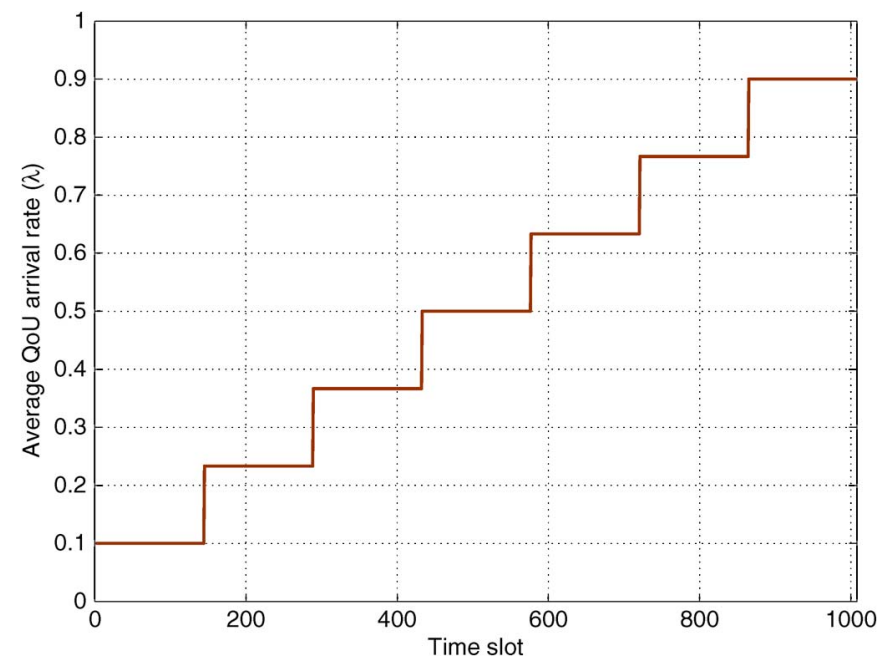

Fig. 12. Average QoU arrival rate following a ramp shape.

It would also be interesting to examine the algorithm under nonstationary QoU arrivals. We set the QoU arrival rate as a ramplike function in this simulation. As shown in Fig. 12, the QoU arrival rate evolves linearly and increases from 0.1 to 0.9 ; each rate lasts for a period of a day. Figs. 13 and 14 show the QoU request blocking probability and virtual queue back$\log$ obtained from the simulation, respectively. The algorithm effectively stabilizes the system and keeps the QoU blocking probability around 0.07 . The proposed algorithm exhibits faster response as time elapses, since a higher average QoU arrival rate triggers more frequent algorithm execution.

We next consider the strict power purchase limitation from the macrogrid, i.e., $E_{\max }$. The other settings are the same as those in Figs. 5 and 6, except that 3\% of the QoU requests exceed the limit $E_{\max }$ and are blocked automatically by the MGCC. The simulation results are plotted in Figs. 15 and 16. We observe that there are some disturbance in the convergence of the QoU blocking probability and larger variation of the queue length, which result from the automatic block operations. However, as time elapses, the average queue length is 


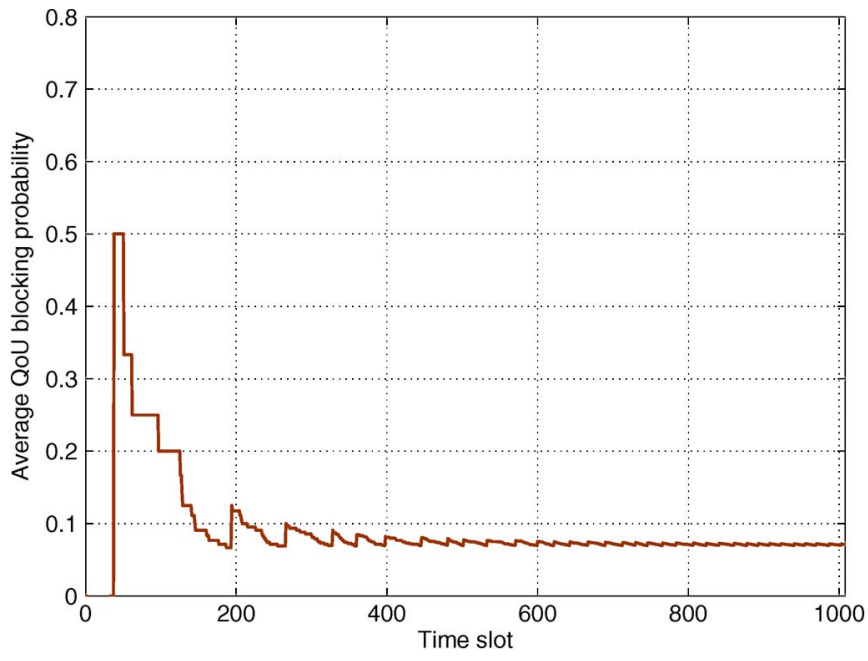

Fig. 13. Average QoU blocking probability with time-varying QoU arrival rates-ramp.

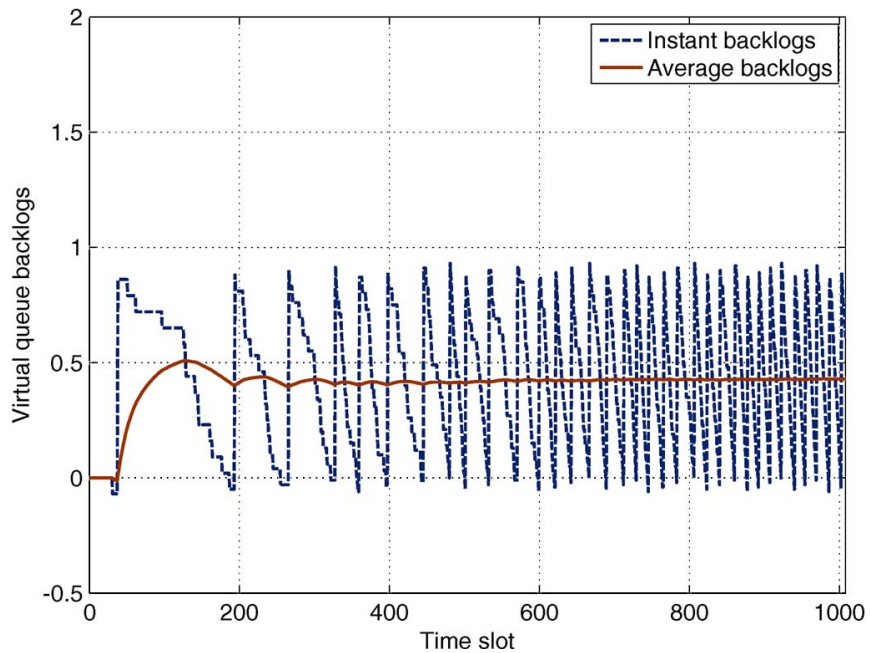

Fig. 14. Instant and average virtual queue backlogs with time-varying QoU arrival rates-ramp.

stabilized, and accordingly, the QoU blocking probability converges to the target value of 0.07 .

We also evaluate a naive policy, which simply blocks arriving QoU requests by tossing a coin with the target probability. Note that this naive policy has a similar computational complexity as the proposed approach. It can be seen in Fig. 17 that the naive coin-tossing policy converges much slower than the proposed adaptive control policy. There is large fluctuation around the target blocking probability. In fact, the proposed scheme converges exponentially, due to the inherent exponential convergence property in Lyapunov-stability-based design [12]. From the control theoretic perspective, the naive policy is actually an open-loop control approach, which may suffer high sensitivity to disturbance and slow convergence. The proposed adaptive control policy is a closed-loop feedback control system, which takes advantage of the feedback of the virtual queue length to achieve disturbance resistance and fast convergence.

Finally, we set $E_{\max }=5$, which means that multiple units of QoU requests may appear in one slot. We keep other parameters as the default setting. The proposed policy is applied to each QoU unit in every slot. We plot the amount of the QoU request

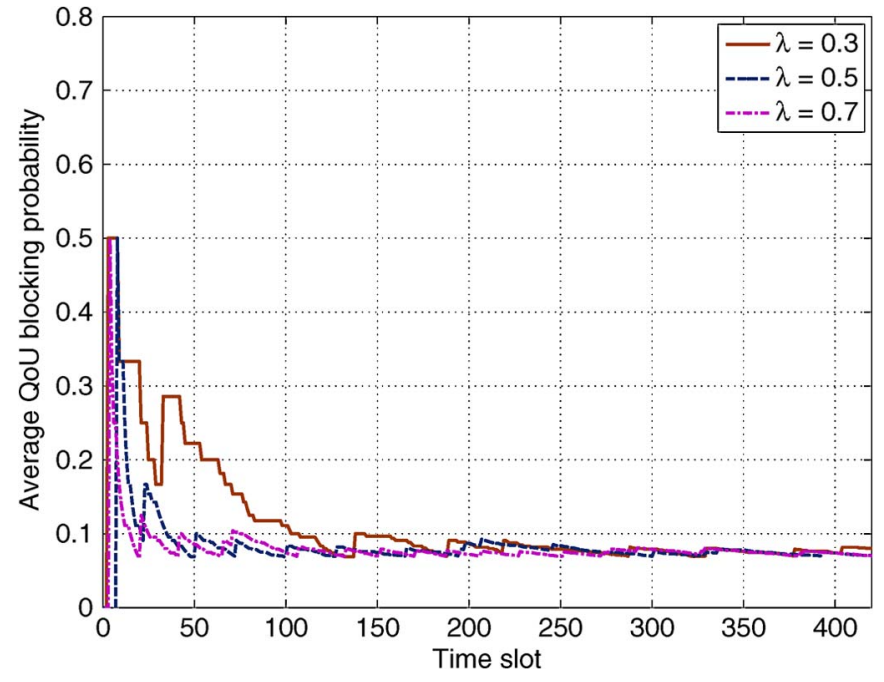

Fig. 15. Average QoU blocking probability under the energy purchase limitation $E_{\max }$.

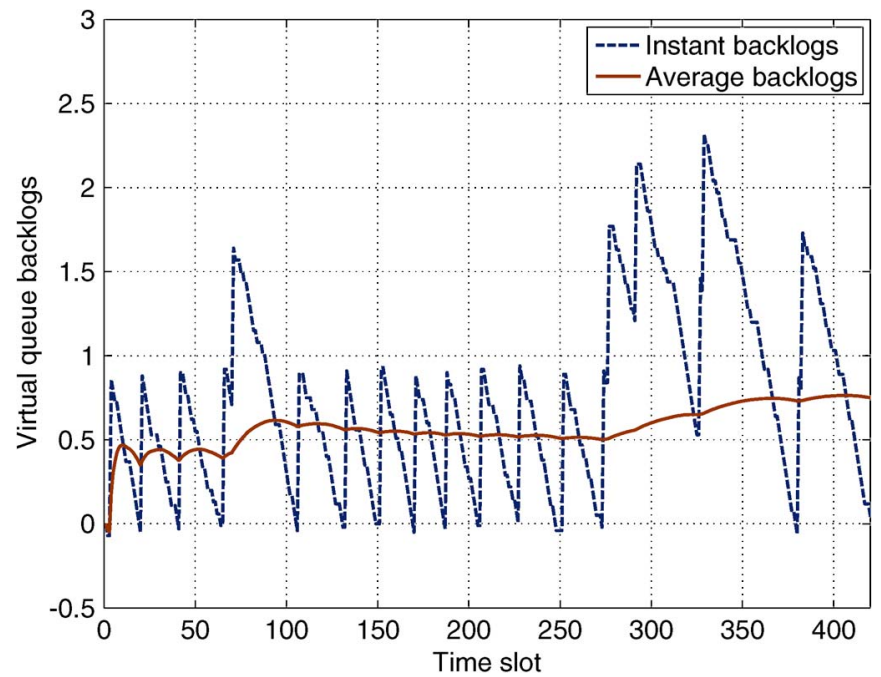

Fig. 16. Instant and average virtual queue backlogs under the energy purchase limitation $E_{\max }$

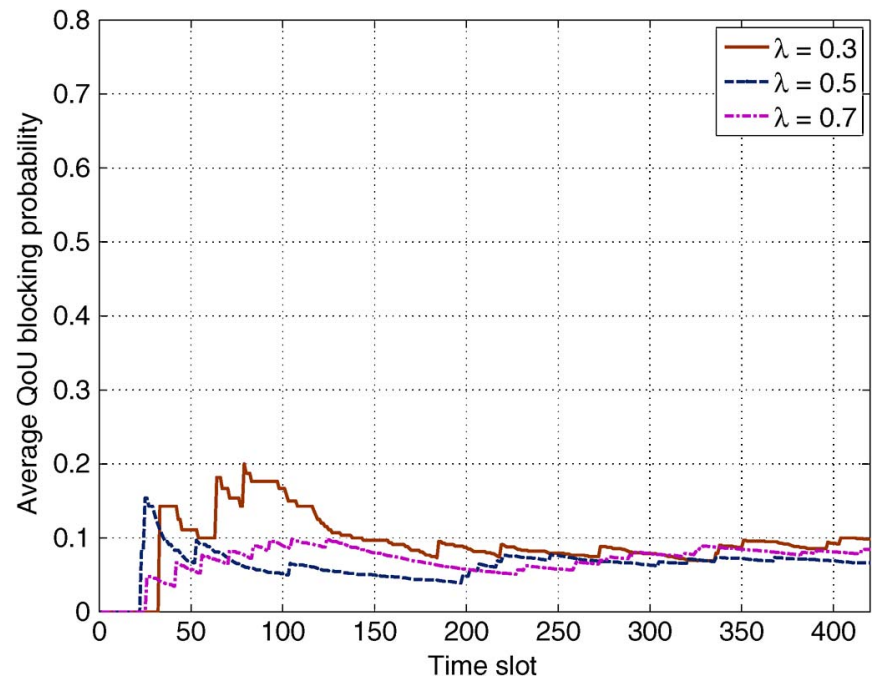

Fig. 17. Average QoU blocking probability with energy purchase limitation and the naive policy. 


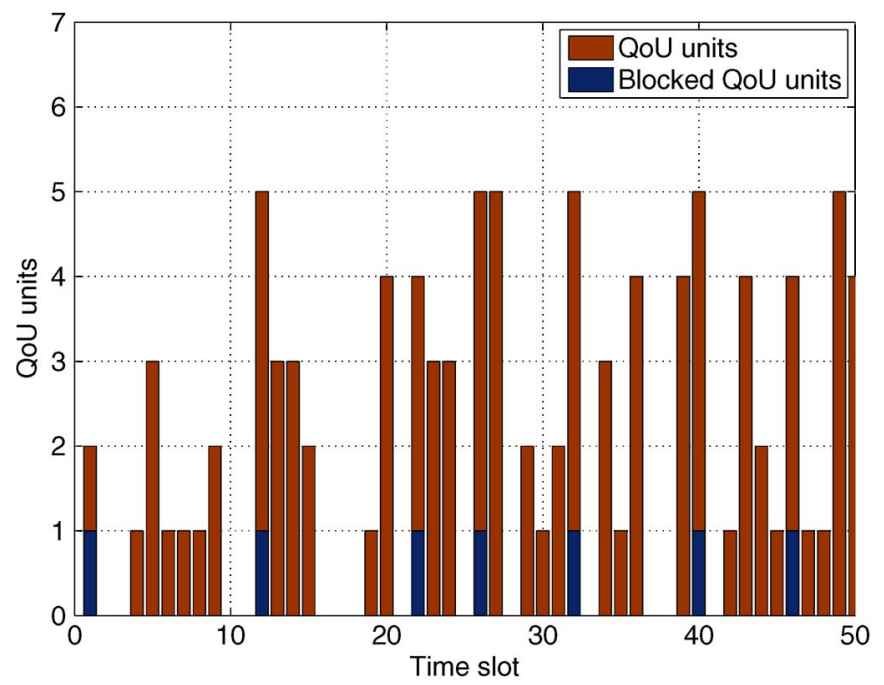

Fig. 18. Total QoU requests and blocked QoU requests when $E_{\max }=5$.

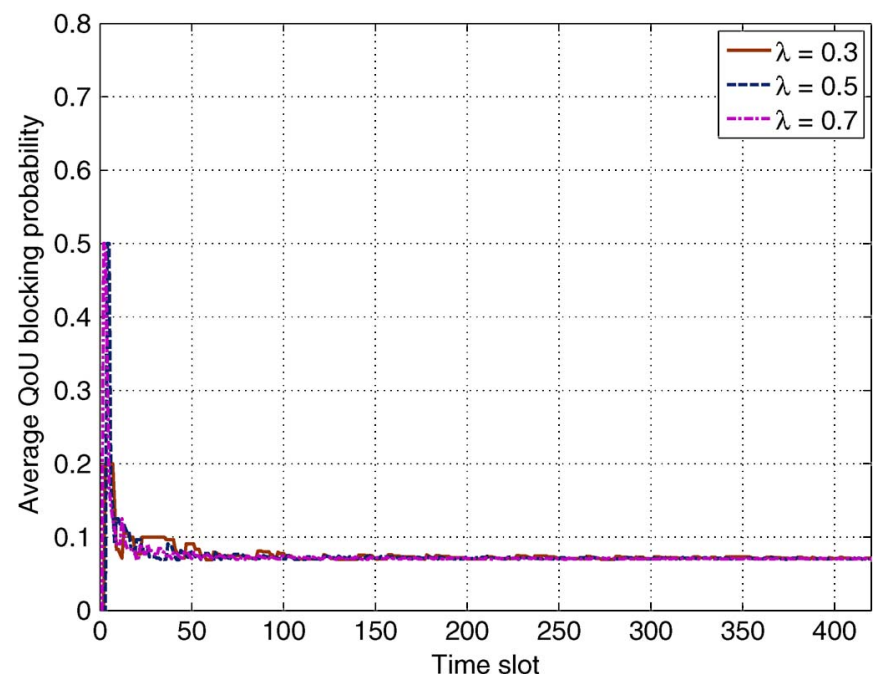

Fig. 19. Average QoU blocking probability when $E_{\max }=5$.

units and the blocked units when $\lambda=0.7$ for the first 50 time slots in Fig. 18. It can be observed that, during the periods that have multiple units of QoU, the proposed algorithm blocks some of the requests for maintaining the target QoU blocking probability. Accordingly, from Figs. 19 and 20, we observe that the average QoU blocking probability is stabilized around 0.07 and the average virtual queue length is also stabilized.

\section{RELATED WORK}

Smart grid is regarded as the next generation power grid with two-way flows of both electricity and information. In smart grid, information technologies and computational intelligence are integrated across electricity generation, transmission, distribution, and consumption to achieve green, reliable, efficient, and sustainable energy goals. We refer interested readers to several comprehensive reviews of smart grid technologies and related research challenges [2], [13], [20]-[22] and the references therein for more details.

Microgrid is a new grid structure to group DRERs and local residents loads, which provides a promising deployment model

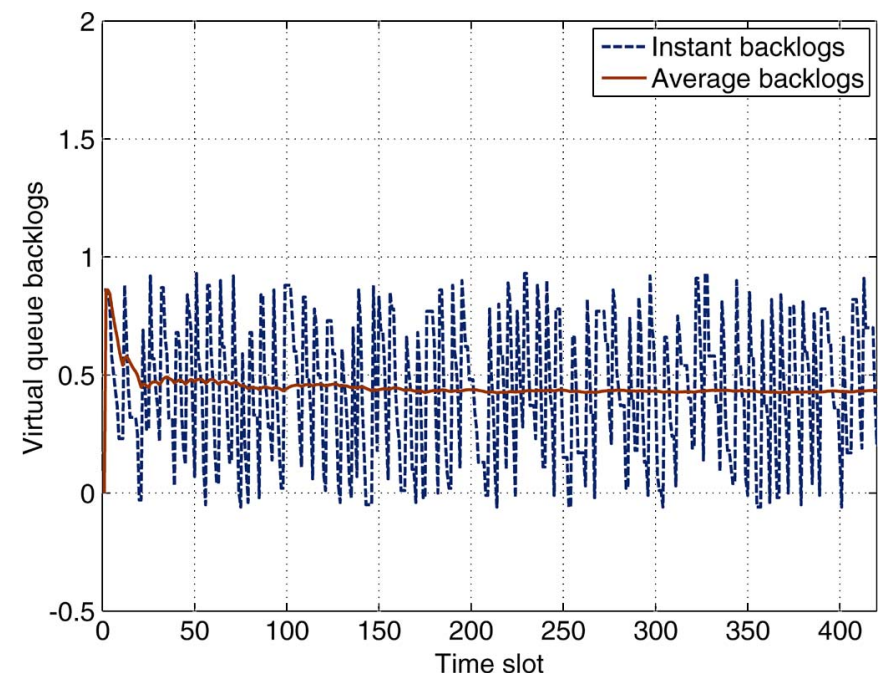

Fig. 20. Instant and average virtual queue backlogs when $E_{\max }=5$.

for the future smart grid. In [3], the authors provided a review of the microgrid structure with distributed energy resources. In [6] and [23], the integration of random wind power generation into the grid for cost-effective operation was investigated. In [24], the authors proposed a useful online method to discover all available DRERs within the island-mode microgrid and compute a good DRER access strategy. In [25], the authors presented an optimal scheduling algorithm to minimize the overall cost of electricity and natural gas for building operation with microgrid technology. The problem of optimal residential demand management was investigated in [15] and [26], corresponding to time-varying energy generation and price and maximizing user benefit. These prior works aimed to balance the time-varying energy generation and user demand, which is one of the most important requirements of microgrid management. In a recent work [27], we incorporate the theory of majorization to develop algorithms for smooth electric power scheduling in power distribution networks.

Lyapunov optimization is a stochastic optimization method [10]. It has been widely used and extended in the communications and networking area [11], [28]-[30]. In [30], Lyapunov optimization was used in the proof of the stability of a rate control algorithm for heterogeneous streaming videos. In two recent works [8], [31], the authors investigated the problem of profit maximization for renewable energy utility companies. The Lyapunov optimization method was applied to jointly optimize the power procurement and dynamic pricing, while meeting consumer requests.

\section{CONCLUSION}

In this paper, we have developed an adaptive QoU control policy for microgrid energy scheduling, while taking into account the stabilization of the QoU block probability. We transformed the QoU scheduling problem to a queue stability problem by introducing a QoU blocking virtual queue and proved that the stability of the virtual queue is equivalent to QoU assurance. We applied the Lyapunov optimization method to solve this problem and developed a minimum weight 
matching algorithm with low complexity and online operation. The stability of the system was proven. Our simulation study showed that the proposed approach is effective and robust for QoU provisioning in microgrids.

\section{REFERENCES}

[1] The Recovery Act, RECOVERY.gov, Washington, DC, USA, Feb. 2009.

[2] X. Fang, S. Misra, G. Xue, and D. Yang, "Smart grid-The new and improved power grid: A survey," IEEE Commun. Surveys Tuts., vol. 14, no. 4, pp. 944-980, 2012.

[3] J. Huang, C. Jiang, and R. Xu, "A review on distributed energy resources and microgrid," Renew. Sustainable Energy Rev., vol. 12, no. 9, pp. $2472-$ 2483, Dec. 2008.

[4] K. Nagothu, B. Kelley, M. Jamshidi, and A. Rajaee, "Persistent Net-AMI for microgrid infrastructure using cognitive radio on cloud data centers," IEEE Syst. J., vol. 6, no. 1, pp. 4-15, Mar. 2012.

[5] A. Papaioannou and F.-N. Pavlidou, "Evaluation of power line communication equipment in home networks," IEEE Syst. J., vol. 3, no. 3, pp. 288294, Sep. 2009.

[6] E. Bitar, K. Poolla, P. Khargonekar, R. Rajagopal, P. Varaiya, and F. Wu, "Selling random wind," in Proc. HICSS, Houston, TX, USA, Jan. 2012, pp. 1931-1937.

[7] S. Shao, M. Pipattanasomporn, and S. Rahman, "Demand response as a load shaping tool in an intelligent grid with electric vehicles," IEEE Trans. Smart Grid, vol. 2, no. 4, pp. 624-631, Dec. 2011.

[8] L. Huang, J. Walrand, and K. Ramchandran, "Optimal power procurement and demand response with quality-of-usage guarantees," Ithaca, NY, USA, arXiv Tech. Rep. [Online]. Available: http://arxiv.org/abs/1112. 0623

[9] A. B. Kukushkin and V. A. Rantsev-Kartinov, "Evidences for and the models of self-similar skeletal structures in fusion devices, severe weather phenomena and space," in Proc. 6th Symp. Current Trends Int. Fus. Res., Washington, DC, USA, Mar. 2005, pp. 1-17.

[10] L. Tassiulas and A. Ephremides, "Stability properties of constrained queueing systems and scheduling policies for maximum throughput in multihop radio networks," IEEE Trans. Autom. Control, vol. 37, no. 12, pp. 1936-1948, Dec. 1992.

[11] M. Neely, E. Modiano, and C. Rohrs, "Dynamic power allocation and routing for time-varying wireless networks," IEEE J. Sel. Areas Commun., vol. 23, no. 1, pp. 89-103, Jan. 2005.

[12] J. Slotine and W. Li, Applied Nonlinear Control. Upper Saddle River, Englewood Cliffs, NJ, USA: Prentice-Hall, 1991.

[13] H. Farhangi, "The path of the smart grid," IEEE Power Energy Mag., vol. 8, no. 1, pp. 18-28, Jan./Feb. 2010.

[14] E. Fumagalli, J. W. Black, I. Vogelsang, and M. Ilic, "Quality of service provision in electric power distribution systems through reliability insurance," IEEE Trans. Power Syst., vol. 19, no. 3, pp. 1286-1293, Aug. 2004.

[15] A. H. Mohsenian-Rad and A. Leon-Garcia, "Optimal residential load control with price prediction in real-time electricity pricing environments," IEEE Smart Grid, vol. 1, no. 2, pp. 120-133, Sep. 2010.

[16] M. J. Neely and R. Urgaonkar, "Opportunism, backpressure, and stochastic optimization with the wireless broadcast advantage," in Proc. Asilomar Conf. Signals, Syst. Comput., Pacific Grove, CA, USA, Oct. 2008, pp. 2152-2158.

[17] K. Astrom and R. Murray, Feedback Systems: An Introduction for Scientists and Engineers. Princeton, NJ, USA: Princeton Univ. Press, 2008.

[18] D. B. West, Introduction to Graph Theory, 2nd ed. Upper Saddle River, NJ, USA: Prentice-Hall, 2000

[19] The National Renewable Energy Laboratory, Western Wind Resources Dataset, Golden, CO, USA. [Online]. Available: http://wind.nrel.gov/ Web_nrel/

[20] S. Amin and B. Wollenberg, "Toward a smart grid: Power delivery for the 21st century," IEEE Power Energy Mag., vol. 3, no. 5, pp. 34-41, Sep./Oct. 2005.

[21] F. Li, W. Qiao, H. Sun, H. Wan, J. Wang, Y. Xia, Z. Xu, and P. Zhang, "Smart transmission grid: Vision and framework," IEEE Trans. Smart Grid, vol. 1, no. 2, pp. 168-177, Sep. 2010.

[22] C. W. Clark, The Smart Grid: Enabling Energy Efficiency and Demand Response. Lilburn, GA, USA: Fairmont Press, 2009.

[23] X. Liu, "Economic load dispatch constrained by wind power availability: A wait-and-see approach," IEEE Trans. Smart Grid, vol. 1, no. 3, pp. 347355, Dec. 2010.

[24] X. Fang, D. Yang, and G. Xue, "Online strategizing distributed renewable energy resource access in islanded microgrids," in Proc. IEEE GLOBECOM, Houston, TX, USA, Dec. 2011, pp. 1931-1937.
[25] X. Guan, Z. Xu, and Q. Jia, "Energy-efficient buildings facilitated by microgrid," IEEE Trans. Smart Grid, vol. 1, no. 3, pp. 243-252, Dec. 2010.

[26] N. Li, L. Chen, and S. H. Low, "Optimal demand response based on utility maximization in power networks," in Proc. IEEE PES Gen. Meeting, Detroit, MI, USA, July 2011, pp. 1-8.

[27] Y. Huang, S. Mao, and R. M. Nelms, "Smooth electric power scheduling in power distribution networks," in Proc. IEEE GLOBECOM-Workshop, Anaheim, CA, USA, Dec. 2012, pp. 1469-1473.

[28] M. J. Neely, "Delay analysis for maximal scheduling with flow control in wireless networks with bursty traffic," IEEE/ACM Trans. Netw., vol. 17, no. 4, pp. 1146-1159, Aug. 2009.

[29] L. Huang and M. J. Neely, "Delay reduction via lagrange multipliers in stochastic network optimization," IEEE Trans. Automat. Control, vol. 56, no. 4, pp. 842-857, Apr. 2011.

[30] Y. Huang, S. Mao, and S. F. Midkiff, "A control-theoretic approach to rate control for streaming videos," IEEE Trans. Multimedia, vol. 11, no. 6 , pp. 1072-1081, Oct. 2009

[31] M. J. Neely, A. S. Tehrani, and A. G. Dimakis, "Efficient algorithms for renewable energy allocation to delay tolerant consumers," in Proc. IEEE SmartGridComm, Oct. 2010, pp. 549-554.

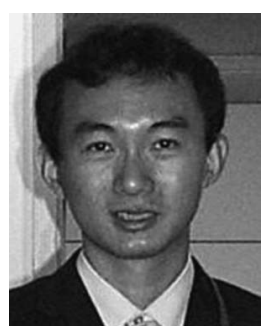

Yingsong Huang (S'12) received the B.S. degree in automation and the M.S. degree in control theory and control engineering from Chongqing University, Chongqing, China. Since 2007, he has been working toward the Ph.D. degree in the Department of Electrical and Computer Engineering, Auburn University, Auburn, AL, USA.

He was a Senior R\&D Engineer with Advantech Corporation Ltd. His research interests include modeling, control, and optimization in smart grid and computer networks.

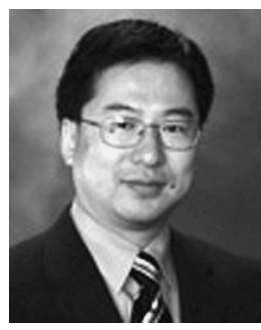

Shiwen Mao (S'99-M'04-SM'09) received the $\mathrm{Ph} . \mathrm{D}$. degree in electrical and computer engineering from Polytechnic University, Brooklyn, NY, USA (now the Polytechnic Institute of New York University), in 2004.

From 1997 to 1998, he was a Research Staff Member with IBM China Research Laboratory. From 2003 to 2006, he was a Postdoctoral Research Associate/Research Scientist with the Bradley Department of Electrical and Computer Engineering, Virginia Polytechnic Institute and State University, Blacksburg, VA, USA. He is currently the McWane Associate Professor with the Department of Electrical and Computer Engineering, Auburn University, Auburn, AL, USA. He is the holder of one U.S. patent. He is a coauthor of TCP/IP Essentials: A Lab-Based Approach (Cambridge University Press, 2004). His research interests include cross-layer optimization of wireless networks and multimedia communications, with current focus on cognitive radio networks, femtocell networks, $60 \mathrm{GHz}$ mmWave networks, and smart grid.

Dr. Mao is on the Editorial Board of IEEE TRANSACTIONS ON WIRELESS COMMUNiCATIONS, IEEE COMMUNICATIONS SURVEYS AND TUTORIALS, Elsevier Ad Hoc Networks Journal, and Wiley International Journal of Communication Systems. He is the Director of E-Letter of the IEEE Communications Society Multimedia Communications Technical Committee. He was a corecipient of the IEEE ICC 2013 Best Paper Award and received the 2013 IEEE ComSoc MMTC Outstanding Leadership Award. He was a recipient of the U.S. National Science Foundation Faculty Early Career Development Award in 2010, the Auburn Alumni Council Research Awards for Excellence-Junior Award in 2011, and two Auburn Author Awards in 2011. He was also a corecipient of The 2004 IEEE Communications Society Leonard G. Abraham Prize in the Field of Communications Systems and The Best Paper Runner-up Award at The Fifth International Conference on Heterogeneous Networking for Quality, Reliability, Security, and Robustness (QShine) in 2008. 